\title{
COMPETITIVIDAD DEL COMERCIO EXTERIOR PERUANO
}

\author{
Peruvian foreign trade competitiveness
}

\author{
Jorge Arroyo Prado ${ }^{1}$
}

PRESENTACIÓN: OCTUBRE 2016

ACEPTACIÓN: NOVIEMBRE 2016

\section{RESUMEN}

En los últimos años, el Perú viene estableciendo convenios de libre comercio con diversos países, pero hasta la fecha lo ha hecho sin una visión clara del mediano y largo plazo con la intervención de los grupos de interés peruanos. En ese sentido, se ha realizado una interpretación de la teoría de Porter y se ha ampliado su sistema denominado diamante, aplicable a países en vías de desarrollo. Con base en este sistema, se propone una política a seguir para el logro de la competitividad del comercio exterior en el Perú.

Palabras clave: Acuerdos, competitividad, visión de mediano y largo plazo, comercio exterior, teoría de Porter

\begin{abstract}
In recent years the Peru has established free trade agreements with countries but without a clear vision of medium and long term with the intervention of Peruvian interest groups. There has been an interpretation of the theory of Porter and expanded his call diamond applicable to developing countries that proposes a policy to be followed to achieve the competitiveness of Peruvian foreign trade.

Keywords: Agreements; competitiveness, vision of medium and long term; foreign trade; Porter's theory
\end{abstract}

1 Facultad de Ingeniería Universidad Ricardo Palma. Lima, Perú E-mail: fac.ingenieria@urp.edu.pe 


\section{EL PORQUÉ DE LA COMPETITIVIDAD}

En 1990, Michael E. Porter publicó La ventaja competitiva de las naciones, libro que tuvo gran impacto en los ambientes académicos, empresariales y gubernamentales de todo el mundo. Buscando determinar cuáles son «los atributos de una nación que fomentan las ventajas competitivas en determinados sectores», el profesor Porter elaboró un modelo que permite comprender el proceso que lleva a lograr ventajas competitivas internacionales y, a la vez, identificar la manera en que los países pueden desarrollar su competitividad, es decir, mejorar la cantidad y calidad de los recursos que poseen y la capacidad para utilizarlos de manera más eficiente. La crítica de sus opositores se centra en el hecho de que este libro tiene como base la investigación, evaluación y resultados con empresas corporativas de países desarrollados.

¿Sería posible que los conceptos de La ventaja competitiva de las naciones puedan aplicarse a un país en vías de desarrollo como el Perú? La respuesta la formularemos intentando adaptar estos conceptos a una visión en la que el comercio exterior peruano tenga como objetivo fundamental el desarrollo sostenible así como su inserción en el comercio global sin dejar de lado las normas ambientales internacionales que contribuyen a lograr el bienestar general de la comunidad peruana.

\section{EL MODELO DE PORTER AMPLIADO}

Según Michael Porter, como ya lo dijimos líneas arriba, la competitividad de un país y sus empresas puede definirse como la capacidad para diseñar, producir y comercializar bienes y servicios mejores -y a buen precio- que los de la competencia internacional. Si se alcanza este logro, se reflejará en una evidente mejora del nivel de vida de la población. De acuerdo con estas características, la idea básica se centra en que la competitividad no se hereda ni depende de la coyuntura económica, sino que hay que crearla con esfuerzo e iniciativa propios.

Lo que hace próspero a un país es la capacidad de sus empresas para alcanzar elevados niveles de productividad, es decir, la capacidad de usar con eficiencia y creatividad la mano de obra, los recursos naturales y el capital, respetando siempre el medio ambiente. En el enfoque de Porter no importa tanto qué y cuántos recursos se poseen, sino qué se hace con los que se tienen. Esto obliga a que las empresas y países en vías de desarrollo que buscan ser competitivos no sean aquellos que descubren un nuevo mercado o la tecnología más adecuada de producción, sino los que implementen o adapten los mejores cambios en la forma más rápida posible, como las normas técnicas y ambientales que los países desarrollados tienen para sus productos.

De acuerdo con Porter, el que una nación cuente con sectores capaces de competir exitosamente en el mercado internacional depende en gran medida del contexto que rodea a las empresas que conforman cada sector, pues estas no son entes aislados.

El diamante de Porter, eje de sus conceptos, se sustenta, en que las

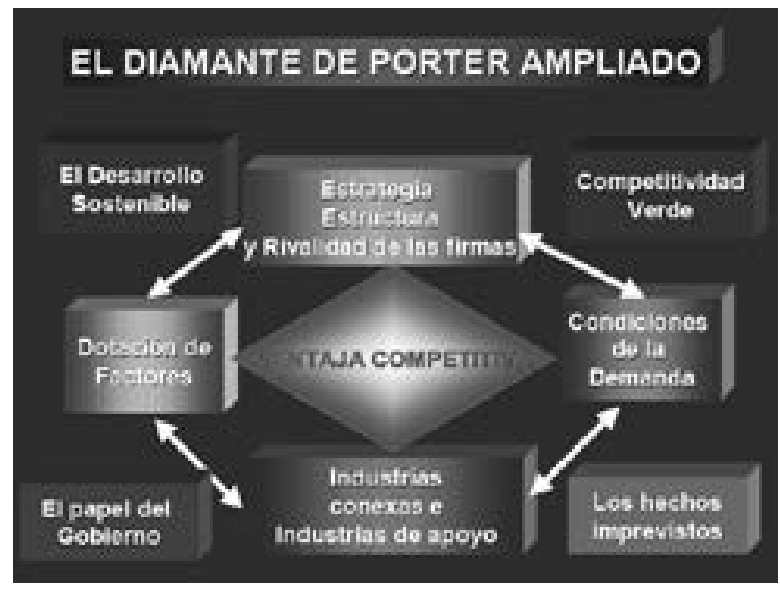

Fig. 1. El diamante de Porter ampliado. 
empresas de un país siempre estarán determinadas por la interacción de cuatro grupos de atributos invariables:

1. La dotación de factores

2. Las condiciones de la demanda

3. Los proveedores, las industrias relacionadas y de apoyo

4. Las estrategias, estructuras y rivalidad de las empresas

Para completar su diamante, en el entorno en que se ubican estas empresas, Porter reconoce la importancia y existencia de dos elementos:

5. Los hechos imprevistos

6. El papel del Gobierno

Sin embargo, para el caso de las empresas del país, sin modificar el diamante de Porter, se le puede complementar al entorno dos elementos adicionales que hagan viable su aplicación a realidades empresariales como la peruana:

7. El desarrollo sostenible

8. La competitividad verde

En este modelo, la base de la competitividad o la capacidad para competir no deriva de los cuatro atributos ni siquiera del conjunto de atributos considerados como un todo, sino de su interrelación, de su reforzamiento mutuo. Esto significa que el efecto que cada uno de ellos pueda causar depende del estado de los otros, que las ventajas de uno puedan crear o perfeccionar ventajas en otros, y que las desventajas de uno puedan acarrear desventajas en los otros. A este concepto de total dinamismo Porter lo denominó el diamante de la competitividad.

Los sectores exitosos en la economía internacional son aquellos a los cuales el diamante de la competitividad les es favorable. Sin embargo, esta afirmación no implica que todas las empresas sean exitosas; más aún, cuanto más competitivo sea el entorno, tanto más probable será que algunas de estas se queden en el camino, ya que no todas tienen iguales habilidades ni explotan similarmente su entorno.

\subsection{La dotación de factores}

El primer atributo del diamante de competitividad hace referencia a la situación del país respecto de lo que comúnmente se llama dotación de los factores (recursos humanos, recursos físicos, conocimientos, capital e infraestructura), es decir, a los inputs necesarios para competir en una industria. Las condiciones de los factores no dependen solo de los factores en sí, sino del grado de eficiencia y efectividad con que se los armonice y dirija hacia el objetivo de producir un bien altamente competitivo.

Por otro lado, no todos los factores tienen la misma importancia para la obtención de la competitividad. Para explicar esta idea, Porter clasifica los factores en básicos y avanzados, por un lado; y en generalizados y especializados, por el otro. Los factores básicos comprenden los recursos naturales, el clima, la situación geográfica, la mano de obra no especializada o poco especializada; y los factores avanzados, la infraestructura, los recursos humanos altamente especializados y el soporte en ciencia y tecnología. Los generalizados, por su parte, son los que pueden aplicarse a varias actividades, mientras los especializados tienen un radio de acción circunscrito, como es el caso de las empresas exportadoras que deben ser calificadas por tener una "producción más limpia” ambientalmente.

Así, tenemos que la visión de una actividad agroexportadora, como en el caso de la agricultura en el Perú, tiene como factores básicos a considerar: las variadas condiciones climatológicas (microclimas) y la disponibilidad de tierras, así como el uso y disponibilidad de mano de obra no capacitada. Los factores avanzados serían los proyectos de irrigación y la infraestructura creada para explotar las tierras. La investigación tecnológica en un campo específico, como los estudios del organismo genéti- 
camente modificado (OGM) usado en el Centro Internacional de la Papa, o el Instituto del Espárrago constituyen factores especializados. Mientras que los factores básicos se heredan o se crean mediante inversiones modestas, los avanzados requieren grandes inversiones y esfuerzos de largo alcance.

En los últimos años, la globalización ha hecho menos esencial la disponibilidad de los factores básicos, pues hay gran movilidad de recursos y las empresas pueden trasladar sus unidades productivas ahí donde estos se encuentren. Hoy, la ubicación geográfica de las plantas industriales no se realiza solo en función de la localización de las materias primas, sino también teniendo en cuenta el lugar donde estas pueden agregar más valor al proceso productivo y proteger al medio ambiente.

A diferencia del Perú, pródigo en recursos naturales, los países asiáticos se vieron obligados a generar competitividad para poder acceder al mercado internacional, y lo lograron. Hoy, el país se enfrenta al reto de evolucionar del viejo modelo rentista, basado en recursos naturales y mano de obra barata, al desarrollo de ventajas competitivas que le permitan una adecuada inserción en la nueva economía mundial. La apertura comercial ofrece muchas oportunidades para beneficiar al medio ambiente, principalmente, mediante acceso a tecnologías limpias, mercados para productos de exportación con mayor valor agregado y, sobre todo, a partir de la estrecha integración con un mundo más preocupado por el tema ambiental.

La mayor parte de los impactos ambientales están relacionados con el crecimiento económico, algo que de todas maneras quisiéramos conseguir con o sin acuerdos comerciales, y para lo cual necesitamos desarrollar nuestras normas y regulaciones ambientales. Es muy probable que el efecto tecnológico favorezca al medio ambiente. La presión competitiva internacional, la globalización de las prácticas empresariales y la inversión extranjera, en general, fomentan un cambio productivo hacia tecnologías y gestiones más modernas y con menores impactos ambientales. La inversión extranjera, muchas veces, estimula la producción limpia en el país en desarrollo adonde llega.

De estos argumentos no se debe concluir que no se requieren normas o regulaciones ambientales. Todo lo contrario, si queremos crecer, necesitamos estas normas y regulaciones a como dé lugar. La pregunta que entonces surge es ¿por qué no utilizar estratégicamente la coyuntura política que ofrece la negociación comercial para mejorar nuestra política ambiental? Existen varios riesgos si queremos seguir esta estrategia. Primero, se corre el peligro de distorsionar nuestras prioridades ambientales. Las iniciativas de política podrían supeditarse a los temas que preocupan a los grupos de interés en el extranjero, incluyendo los grupos proteccionistas, cuyas prioridades pueden ser diferentes a las nuestras. Segundo, al entrar en este juego, se estaría reconociendo tácitamente que el medio ambiente y el comercio internacional están íntimamente relacionados, asunto que hace más difícil resistir las presiones por incluir condiciones o salvaguardas "ambientales" en el tratado mismo.

\subsection{Las condiciones de la demanda}

Por condiciones de la demanda se entiende la composición, el tamaño, ritmo de crecimiento y grado de sofisticación de las empresas en el mercado nacional, puesto que al estimularlas a la mejora y a la innovación, estas se convierten en determinantes de la competitividad. Respecto de la composición, un mercado interno segmentado, de compradores exigentes y de necesidades precursoras, puede presionar a las empresas al constante perfeccionamiento, que puede reflejarse, por ejemplo, al tener una producción limpia.

En cuanto al tamaño y el ritmo de crecimiento del mercado, las economías de escala que justifican la inversión en investigación y desarrollo pueden impulsar la competitividad al incentivar las inversiones. Sin embargo, Porter también seńala que un mercado interno pequeńo, como sería el caso del Perú, puede convertirse en un incentivo para esforzarse por competir en el mercado externo. 
Por último, la sofisticación de los compradores o su internacionalización se refiere a todo aquello que identifique y transmita sus gustos o hábitos de consumo en los productos que adquiere y que se les puede ofrecer. En el caso peruano, dado el escaso poder adquisitivo y sin mayores exigencias del consumidor local, la demanda para muchos productos viene dada por el mercado externo, como sucede con los espárragos y el palmito, los que se exportan cumpliendo las normas ambientales a diversos mercados internacionales.

\subsection{Los proveedores, las industrias relacionadas y de apoyo}

En el modelo de Porter, la competitividad de un sector se sustenta en la disponibilidad de las industrias o proveedores conexos que, a su vez, sean por sí mismos competitivos. Las industrias relacionadas o conexas comprenden a todas aquellas que comparten tecnologías comunes, insumos y productos complementarios, es decir, aquellas con las que las empresas pueden compartir o coordinar actividades de su cadena de valor, como el desarrollo de tecnología, fabricación, distribución, comercialización o servicio de producto.

Normalmente, en los países en desarrollo, dada la falta de proveedores competitivos, las industrias aparecen como sectores aislados en vez de clusters o industrias relacionadas. Esto obliga a que la mayoría de los componentes tengan que importarse y a que las empresas se vean forzadas a procesos de integración vertical. Algunas veces, como no es posible obtener los insumos requeridos localmente, las industrias se ven obligadas a dedicarse a actividades que no forman parte de su negocio principal, como es el caso de la generación de electricidad o la operación de transporte en el sector minero peruano. En el Perú, la presencia en el mercado de gran número de pequeñas empresas y microempresas, caracterizadas por su flexibilidad, obliga a replantear el reto de cómo integrarlas en el circuito de la cadena productiva para constituir clusters de empresas a fin de lograr competitividad.

\subsection{Las estrategias, estructuras y rivalidad de las empresas}

El último determinante de la ventaja competitiva de un país, según Porter, es el contexto en el que se crean, organizan y gestionan sus empresas exportadoras, así como la naturaleza de la rivalidad entre ellas. Parte de este contexto deriva del hecho de que, en el ámbito nacional, existen prácticas y enfoques de gestión comunes a las empresas. Porter menciona, entre otras prácticas, la formación y orientación de los líderes, el peso de la iniciativa individual, los medios para la toma de decisiones, la actitud hacia las actividades internacionales y la relación entre los trabajadores y los directivos.

De la misma manera, la idiosincrasia de una nación influye sobre las formas de organizar y gestionar las empresas y, de alguna manera, condiciona sus posibilidades para lograr ventajas competitivas. Los objetivos de las empresas y de las personas juegan también un papel importante, como pueden ser las motivaciones y los compromisos. En cuanto a la rivalidad interna o doméstica de las empresas, Porter señala que la creación y persistencia de la ventaja competitiva en un sector determinado están asociadas a una intensa rivalidad doméstica. La competencia en el mercado de origen sirve de estímulo a las empresas para que mejoren la calidad de sus productos o servicios, reduzcan precios e innoven.

En el caso peruano, el cambio hacia un entorno más competitivo se inicia en 1990. La apertura y la liberalización de los mercados, la eliminación de las barreras de entrada y salida a los productos, así como la asunción de un rol menos protagónico del Estado, además de todo un trabajo complementario que evitó las prácticas anticompetitivas y los abusos frente a los consumidores, fueron las condiciones necesarias para crear un mercado competitivo. 
Sin duda alguna, el cambio hacia una cultura de mercado y hacia la internalización de las nuevas reglas de juego, entre ellas las ambeintales, por parte de las diferentes empresas exportadoras no es tarea fácil. Algunos sostienen que es relativamente más fácil abrir los mercados que abrir las mentes, y que incentivar nuevas actividades y actitudes es verdaderamente una tarea compleja que precisa de un cambio generacional y de una reforma de la educación.

\subsection{Los hechos imprevistos}

Los hechos imprevistos suponen uno de los factores adicionales que complementan el diamante de la competitividad. Precisamente, este fue uno de los hallazgos del estudio empírico de Porter. Los acontecimientos imprevistos también ejercen influencia sobre la ventaja competitiva. Se dice que son los incidentes ajenos a una nación y sobre los cuales las empresas o el Gobierno no tienen mayor control.

La importancia de los hechos imprevistos para las ventajas competitivas radica en el hecho de que pueden alterar la posición competitiva de las naciones. Sin embargo, el que un hecho imprevisto se convierta en ventaja competitiva depende del diamante nacional. Porter hace ver que, inclusive, lo que puede parecer fruto de lo imprevisto es, en realidad, resultado de las diferencias de los entornos nacionales.

Entre estos hechos Porter menciona los inventos, el talento creativo o recreativo de las personas, las discontinuidades tecnológicas, las discontinuidades en los costos de los insumos, los cambios significativos en los mercados financieros mundiales o en el tipo de cambio, los aumentos insospechados de la demanda mundial o regional, las decisiones políticas de gobiernos extranjeros y los conflictos bélicos, las convulsiones sociales o las acciones de terrorismo. Otros hechos imprevistos que repercuten sobre el diamante nacional pueden ser también los desastres naturales. Un ejemplo es el Fenómeno de El Niño, que afecta el entorno del Perú con desastres de diversa índole. Los paros y huelgas inesperadas y algunos fenómenos de la naturaleza suponen, asimismo, hechos impresvistos a lo que debemos hacerles frente.

\subsection{El papel del Gobierno}

Este es el otro factor adicional que completa el modelo de Porter y al que se le otorga gran importancia: el poder que tiene el Gobierno de influir sobre el diamante a través de leyes, normas y políticas, las cuales afectan a cada uno de los determinantes de la competitividad.

Puesto que establece las reglas de juego, el Gobierno puede mejorar o deteriorar la ventaja nacional o alterar el clima nacional para la competitividad. Porter sostiene que si bien es tentadora la idea de colocar al Gobierno como el sexto determinante del diamante, en realidad su papel en la competencia internacional es el de influir positiva o negativamente sobre los cuatro determinantes. Más aún, Porter advierte sobre el peligro de perder de vista el hecho de que la influencia del Gobierno sobre la ventaja competitiva es inevitablemente parcial: puede aumentar o disminuir las probabilidades de conseguir ventaja competitiva, pero no puede crearla, porque esta es tarea exclusiva de las empresas exportadoras.

Desde la perspectiva del modelo porteriano, se acepta que el papel del Gobierno es proporcionar el marco propicio para un entorno favorable, es decir, actuar como agente promotor y responsable de dotar al país de infraestructura básica, desarrollo tecnológico y servicios básicos de educación y salud para permitir a las empresas competir con ventaja en los mercados internacionales. Un aspecto que se debe tener en cuenta y que es de gran importancia en el actual contexto latinoamericano, es saber que no basta con declarar la economía de mercado para que esta funcione. Hace falta también el adecuado 
funcionamiento de las instituciones gubernamentales, con reglas de juego definidas y un Estado eficaz y sin corrupción.

Una herramienta básica en los negocios internacionales para determinar cuán riesgoso o atractivo es un país como destino de inversiones es el denominado riesgo país. El efecto del aumento del riesgo país es la exigencia de un mayor retorno o tasa de descuento para los proyectos de inversión. Ello significa que si el riesgo país es alto, entonces muchas inversiones dejarán de implementarse o se llevarán a cabo solo aquellas que permitan una rápida recuperación del capital.

Un elemento importante del riesgo país es el riesgo político institucional. Para el inversionista es fundamental saber si en el país se aplican las leyes, si las decisiones judiciales son independientes e imparciales y si la administración de justicia es confiable; es decir, si se garantiza y respeta el marco institucional del país. En ese sentido, el adecuado funcionamiento de las instituciones genera un entorno jurídico confiable para las inversiones. Sin un óptimo sistema proveedor de seguridad jurídica para todos, el mercado no podría funcionar eficientemente.

\subsection{El desarrollo sostenible}

Si es que los países en vías de desarrollo buscan que sus empresas alcancen ventajas competitivas dentro de los conceptos que plantea Porter, tienen que tener en cuenta cómo las decisiones políticas de los Gobiernos, actualmente, han incorporado la idea precursora de lograr un desarrollo sostenible y, dentro de esa misma idea, la consideración de que las empresas exportadoras contribuyen a lograr el bienestar nacional.

Se supone que este enfoque parte de la existencia de una conciencia pública que ha integrado en sus decisiones los aspectos económicos, sociales y ambientales en los que la preocupación ambiental está arraigada a la sociedad y prevalecen los criterios de solidaridad social y equidad generacional. El desarrollo se plantea basado en la consolidación de la democracia con formas de participación ciudadana. Así, la gobernabilidad evolucionaría hasta lograr un sistema en el cual se tenga control sobre las decisiones socioeconómicas y ambientales. De este modo, el mercado seguiría siendo el principal mecanismo de asignación de los recursos, pero los precios incorporarían los costos sociales y ambientales. Las políticas de desarrollo se diseñarían sobre la base de una gama más amplia de herramientas económicas, y estarían basadas en la producción de bienes y servicios con valor agregado. Asimismo, se estrecharían con mayor dinamismo los vínculos comerciales con otros países desarrollados.

También, se desarrollaría una cultura de prevención del deterioro ambiental y se produciría un cambio en los patrones de consumo, ya que el consumidor preferiría bienes y servicios producidos sobre la base de procesos productivos ambientalmente sostenibles. De esta manera, se mejoraría el ingreso de los productores y la calidad de vida de la población. Igualmente, la innovación tecnológica respondería a la nueva demanda por tecnologías eficientes y limpias. Además, se promovería el desarrollo de tecnologías propias sobre la base de la adaptación de las extranjeras. También, se produciría una revalorización del conocimiento tradicional. De esta manera, las nuevas tecnologías y el aprovechamiento de los conocimientos tradicionales se orientarían a solucionar los problemas de pobreza y deterioro ambiental, lo que permitiría desarrollar nuevas actividades económicas sostenibles.

El Gobierno emitiría normas que busquen la eficiencia con la mayor equidad posible, lo que contribuiría a mejorar la gobernabilidad del país. Las relaciones entre el Estado, el sector privado y la sociedad civil se llevarían a cabo de una forma transparente, lo que mejoraría los mecanismos de control social. 


\subsection{La competitividad verde}

Si las empresas exportadoras del país asumen que el desarrollo sostenible es un objetivo común entre todos los países del planeta, encontrarán que, en los llamados países desarrollados, al tener diseńadas y en aplicación determinadas políticas y normas de protección ambiental para sus empresas, ligadas al desarrollo sostenible de su nación, estos gozan una ventaja competitiva que, por ser estrictamente ambiental, la denominamos competitividad verde.

Aunque se podría argumentar que la competitividad verde podría ser también un componente de los factores avanzados del diamante de Porter, consideramos que se debe aceptar el hecho de que las empresas de los países que ya cuentan con infraestructura adecuada para manufacturar productos mediante una producción "limpia" — en base a normas y estándares ambientales establecidos — se encuentran en ventaja competitiva con aquellas empresas de las naciones en donde todavía no se han adoptado estas políticas y normas ambientales. Por ello, las organizaciones de infraestructura adecuada, al verse frente a una competencia de productos que ingresan a su país y que no cumplen con las calidades y condiciones de producción ambiental, en defensa de sus consumidores y productores, exigirán el cumplimiento de las mismas.

Las empresas extranjeras de países desarrollados que se instalan en un país como en el Perú llegan con exigencias ambientales propias que son más avanzadas que las previstas en la legislación del país. Muchas veces, debido a la transferencia tecnológica, y por el efecto demostración, se logra una producción "más limpia". Su trascendencia en el plano de la gestión ambiental nacional puede tener un efecto positivo. Un ejemplo, en ese sentido, es el del sector minero, donde las compañías mineras extranjeras aplican medidas y normas ambientales más exigentes que las que quisiera cumplir el empresario minero nacional.

Sin embargo, para el caso de las empresas exportadoras de otros sectores productivos, surge la necesidad de diseñar y elaborar sus productos siguiendo las normas ambientales de los países en los que tienen sus mercados para tener una aceptación que facilite el negocio. En las negociaciones comerciales con el país receptor, este exigirá el cumplimiento de dichas normas para ingresar a su mercado. Si esta petición no se atiende previsoramente, no solo supondrá una desventaja competitiva sino que, además, se transformará en un encubierto instrumento para-arancelario -IPA- al que hemos denominado Verde por su relación con el medio ambiente.

\section{UNA VISIÓN DE BASES PARA UN COMERCIO EXTERIOR COMPETITIVO}

Lo descrito anteriormente nos permite tener una visión más clara de qué queremos ser y cómo lo lograremos, siempre con miras a un comercio exterior exitoso. Esto es importante, en especial, para las empresas exportadoras: si no contamos con una visión clara, no hay forma de saber adónde nos estamos dirigiendo.

La visión es un término que resulta de gran importancia en la elaboración de una estrategia nacional. Una visión se plasma en un conjunto de planes o acciones que nos llevan a una decisión óptima en un ámbito geográfico. Los planes estratégicos nos dan las pautas concretas que permiten hacer realidad la "visión" planteada para el país. Asimismo, es importante resaltar que la visión debe ser entendida y compartida por todos los ciudadanos.

De nada sirve que solo burócratas calificados de "alto nivel" expliquen su visión si los actores sociales, como en este caso las empresas exportadoras, no la sienten como suya. La visión debe gestarse en la base de las empresas exportadoras de los sectores productivos a fin de cohesionarlos en su conjunto para la acción. La única manera de tener éxito y poder afrontar los retos del futuro es cuidando que 
los líderes empresariales y políticos hagan realidad una visión compartida con participación de toda la población. Esto implica el compromiso de todos los agentes económicos y políticos para concentrar los esfuerzos en la misma dirección.

La visión debe reflejar los valores, creencias y conductas de todos los agentes económico-sociales del país. Por ello, a veces es necesario cambiar algunos de estos valores y conductas con miras a la búsqueda de una nueva "cultura de competitividad". Las ventajas de contar con una visión de futuro compartida son muchas. Entre las más importantes destacan las siguientes:

- Situarse adecuadamente en el mundo, es decir, analizar y entender el contexto internacional en el cual estamos insertos, y definir cómo nos vamos a insertar mejor.

- Adoptar una perspectiva y una actitud de mediano y largo plazo, y así dejar de lado el excesivo sesgo en el análisis de corto plazo.

- Utilizar adecuadamente todos los recursos disponibles: físicos, naturales, humanos, tecnológicos, económico-financieros, políticos, diplomáticos, militares y culturales, en el marco de un desarrollo sustentable, a fin de hacer realidad la visión planteada.

- Formar un sentido de identidad nacional y reforzar la autoestima, de modo que permita a una sociedad definirse en una dirección positiva.

El tener una visión nos da una referencia de la verdadera dimensión de la tarea del desarrollo. Tal vez lo más importante es entender que una visión compartida lleva al compromiso de todas las personas involucradas y a la toma de conciencia de que todos necesitan de todos.

De otro lado, la metodología del diamante de competitividad de Porter ampliado ayuda a gestar la visión compartida para cada actor que participa en el comercio exterior. Los mismos elementos que determinan las ventajas competitivas de una nación del diamante de Porter ampliado son válidos y aplicables al caso del comercio exterior y de las empresas exportadoras del Perú, ya que, en estos casos, la ventaja competitiva surge de la interacción entre las condiciones nacionales y locales. El nuevo tratamiento de la competitividad parte del cambio de las actitudes mentales de los líderes. Solo una población con una actitud competitiva, que comprende la nueva dinámica de la economía mundial en la que los recursos naturales y la mano de obra barata ya no son lo más importante para competir en el mundo, es la que va a permitir desarrollar el potencial del comercio exterior.

La forma de actuar de las personas obedece a su experiencia pasada, a sus creencias, al contexto en el cual ven su mundo, pero muchas veces este no responde a la realidad, y por ello el éxito de ayer muchas veces se convierte en el fracaso de hoy. Por ejemplo, durante muchos años, los empresarios peruanos trabajaron en una economía cerrada, protegida e inestable, donde el Estado era el estratega empresarial. En ese contexto, la información y los lobbies eran la clave del éxito de las empresas, y la estrategia era el bajo costo, por lo que era válido buscar la máxima rentabilidad, ya sea en las relaciones con los clientes o con los proveedores de los recursos. El resultado fue la obtención de rentabilidad, pero con poca innovación de los productos.

Posteriormente, cuando se abrió la economía y se terminaron los subsidios y las tasas de cambio preferenciales, los resultados fueron baja rentabilidad y la pérdida del mercado interno. Los empresarios no estaban preparados para la innovación y la competitividad, donde la clave del éxito es trabajar con una estrategia de alianzas con proveedores, distribuidores y clientes, es decir, compartiendo información. Los empresarios seguían trabajando con los esquemas del pasado, los cuales, en el nuevo contexto, ya no eran válidos. Por ello, el tránsito o cambio del "éxito" al "fracaso" fue un hecho evidente; es decir, el éxito de ayer se convirtió en el causante del fracaso de hoy.

Lo más evidente es que el pasado ya no sirve para predecir el futuro, pero lo más grave es que nos distrae o confunde sobre lo que es verdaderamente relevante en un mundo globalizado y de grandes cambios tecnológicos como el de hoy en día. No se deb perder de vista que la base de una economía 
de mercado es la creación de riqueza. Paradójicamente, en muchos casos, esta resulta siendo mal vista y no aceptada.

Hay variables culturales que llevan a una confrontación entre la riqueza y la pobreza. Tal vez, el hecho de que históricamente la riqueza haya estado, en muchos casos, ligada a la explotación o a la corrupción lleva a no tener un buen concepto sobre ella. En este contexto, el cambio tiene que producirse, y la riqueza obtenida con trabajo, esfuerzo y creatividad tiene que ser bienvenida. El país necesita de creadores de riqueza, que constituye la base de la competitividad dentro del marco de un desarrollo sustentable. Un esquema mental muy difundido es que no solo estamos insatisfechos con lo que somos, sino que tampoco deseamos que los demás sean exitosos. El éxito resulta castigado y, en muchas organizaciones, es mal visto, y no se permite sobresalir o destacar tanto al empresario como al líder.

\section{EL PAPEL DEL GOBIERNO EN ESTA VISIÓN}

Las tareas pendientes del Gobierno como agente facilitador y habilitador de condiciones favorables para la competitividad en el comercio exterior son las siguientes:

- Facilitar y propiciar el desarrollo de la denominada "cultura de la competitividad" a fin de que sean los mismos agentes económicos quienes tomen la decisión y construyan una economía competitiva en el marco del desarrollo sustentable y de protección ambiental.

- Proporcionar una visión positiva de lo que se debe hacer como país con un mensaje coherente y consistente hacia el futuro.

- Proporcionar las reglas de juego claras que permitan abrir un consenso sobre las líneas maestras de la economía del país.

- Mejorar la calidad y la disponibilidad de la infraestructura de servicios básicos para el logro de la competitividad.

- Proporcionar una adecuada seguridad jurídica y mejorar el buen desempeño de las instituciones que faciliten el desarrollo de la competitividad.

- Generar un ambiente que desarrolle la innovación y propicie una mayor competencia de la economía en general mediante incentivos a la inversión para la formación y la capacitación.

- Eliminar definitivamente la corrupción, la delincuencia y concretar un ambiente de seguridad ciudadana.

\section{REFERENCIAS}

[1] Bryant H-Mehrtnst (1996). La cuarta ola. El mundo de los negocios en el siglo XXI. España: Editorial Gránica.

[2] Daniels-Radebaugh-Sullivan (2013). Negocios Internacionales. Ambientes y Operaciones. México: Editorial Pearson.

[3] Hill Ch. (2015). Negocios Internacionales. Cómo competir en el mercado global. México: Editorial McGraw Hill.

[4] Guisado, M. (2002). Internacionalización de la Empresa. Estrategias de entrada en los Mercados Extranjeros. España- Ediciones Pirámide. 